\title{
LOS ARREGLOS INTERBANCARIOS DURANTE EL FRANQUISMO
}

JOSÉ LUIS GARCÍA RUIZ

Universidad Complutense de Madrid

RESUMEN

Una abundante literatura ha insistido en el carácter oligopolistico de la banca española durante el franquismo, fenómeno que habría sido liderado por las grandes instituciones con consecuencias indeseables sobre la oferta de crédito. Además, el oligopolio habría contado con el beneplácito de las autoridades. Sin embargo, el hallazgo de nueva documentación relacionada con los acuerdos interbancarios muestra que los procesos de colusión estuvieron llenos de dificultades e incumplimientos, y que el comportamiento de las autoridades gubernamentales no siempre respondió a los deseos de la gran banca.

\section{ABSTRACT}

An abundant literature has insisted on the oligopolistic character of the Spanish banking system during the Francoism, a phenomenon led by the great institutions with undesirable consequences on the credit supply. In addition, oligopoly would have been backed by the authorities. Nevertheless, new documentation from bank's archives shows that collusion was full of difficulties and breaches, and also that the behaviour of the Government not always responded to the demand of the big banks.

JEL Classification: N24, G21.

$N$. de E.: Fecha de recepción del artículo en la Revista de Historia Económica: mayo, 2001.

Fecha de aprobación del artículo por el Consejo de Redacción: mayo, 2002. 


\section{INTRODUCCIÓN *}

Existe una literatura abundante que ha insistido en el carácter oligopolístico de la banca española durante el franquismo ${ }^{1}$. Según esa literatura, se habría dado un entrecruzamiento de intereses entre un grupo reducido de grandes bancos con el objeto de eliminar la competencia y asegurar un margen financiero remunerador. Los resultados habrían sido un mayor coste del crédito y un empuje a la inflación.

El oligopolio bancario tendría varios aspectos. Por un lado, se basaría en estrategias de cartelización del sector (reparto del mercado, acuerdos de precios) y, por otro, en participaciones cruzadas y consejeros comunes entre las propias entidades bancarias y de éstas con las principales empresas españolas. Este último aspecto ha sido exhaustivamente analizado por los investigadores, por ser fácilmente detectable, aunque no debemos olvidar la atinada crítica que le hizo el banquero Rafael Termes al señalar que el enfoque es muchas veces espurio, ya que la alta gestión nunca pasa por los consejeros comunes; citando al presidente de un gran banco, Termes recordaba que en el Consejo de Administración se sabe lo que ha pasado, en el Comité Ejecutivo lo que está pasando y sólo la alta dirección (presidentes, consejeros delegados o, en su caso, directores generales) conoce «lo que va a pasar» ${ }^{2}$.

Menos conocidas son las estrategias de cartelización, que sólo podrán abordarse satisfactoriamente cuando la historia empresarial de la banca española esté más desarrollada. El trabajo más importante en esta línea es el de Pons (2001), donde mediante procedimientos estimativos indirectos se concluye que los acuerdos de precios no fueron respetados en grado suficiente, por lo que se puede decir que durante el franquismo hubo más competencia vía precio, especialmente en el mercado de captación de depósitos, de lo que hasta ahora se había supuesto.

* Este trabajo es resultado de la participación de su autor en el proyecto de investigación 06/01/23/01 ( Banca y desarrollo económico en Madrid durante el siglo xX»), financiado por la Comunidad de Madrid y dirigido por Gabriel Tortella. Le agradezco a él, a M." Ángeles Pons y a tres evaluadores anónimos las útiles recomendaciones que me han hecho. Eso sí, los posibles errores son sólo míos.

1 Véase Muñoz (1969) y la literatura que ahí se cita. Una aproximación reciente en esta línea es la de Pérez (1997). Con Martín Aceña y Pons (1994) se inicia la revisión de la historia bancaria del franquismo. En Tortella y García Ruiz (2000) y Tortella y García Ruiz (2001) se cuestiona abiertamente la relación de la banca con las autoridades durante el primer franquismo. Nuevas aportaciones son las de Tedde (2001) y Pons (2002).

2 Véase Termes (1991), volumen 2, p. 1684. 
En este trabajo se pretende aportar algo más de luz sobre los acuerdos de precios, conocidos como «arreglos», a partir de fuentes primarias nunca antes utilizadas. Por un lado, se presenta y analiza la documentación de los acuerdos que recibió la Dirección General de Banca y Bolsa, pues tras la Ley de 27 de agosto de 1938 toda la política crediticia quedó bajo la autoridad del Ministerio de Hacienda. Esta documentación se encuentra en el Archivo Central de Hacienda $(\mathrm{ACH})$ y no ha sido hasta ahora utilizada. Por otro lado, se ofrece evidencia empírica de los «extratipos» (tipos de interés pasivos por encima de lo acordado) ofrecidos por una de las entidades líderes, el Banco Hispano Americano, en base a un trabajo inédito realizado con fuentes internas ${ }^{3}$.

\section{EL ARREGLO MODERADOR DE LA COMPETENCIA BANCARIA DE 1941}

Durante la Guerra Civil se rompió la autorregulación de la banca en materia de tipos de interés que había estado vigente desde la Ley de Ordenación Bancaria de 1921. La citada Ley de agosto de 1938 puso este y todos los demás aspectos de la política crediticia bajo el mando del Ministerio de Hacienda, dictándose el 25 de noviembre unos tipos de interés máximos para las operaciones pasivas de bancos y cajas de ahorros, que se completaban con las tarifas de comisiones aprobadas el 15 de enero de 1936. Las comisiones eran el único elemento de coste de las operaciones bancarias que había recibido con anterioridad (desde 1927) una intervención por parte del Estado, refrendando siempre lo dispuesto por el Consejo Superior Bancario, a pesar de que el artículo 4. ${ }^{\circ}$ de la Ley de 1921 indicaba que este Consejo también estaba facultado para «señalar el tipo máximo de interés abonable a las cuentas corrientes, imposiciones, bonos y demás conceptos similares» ${ }^{4}$.

${ }^{3}$ Tortella y García Ruiz (1999). Básicamente, la parte correspondiente al Banco Central fue desarrollada por el primer autor, mientras que la concerniente al Banco Hispano Americano recayó en el segundo.

${ }^{4} \mathrm{La}$ Orden de 25 de noviembre de 1938 fue calificada de «política nefasta» por Pablo Garnica, presidente del Banco Español de Crédito, ya que se había impuesto el criterio gubernamental frente al del Comité Central de la Banca, que, reunido en Burgos el 29 de marzo, había acordado tipos máximos más bajos (de 0,5 por 100 en cuenta corriente, 2 por 100 en cuentas de ahorro, entre 1,5 y 2,5 por 100 en imposiciones a plazo fijo). Véanse Actas del Consejo de Administración del Banco Español de Crédito, 19 de noviembre de 1937 y 18 de enero, 28 de marzo, 22 de mayo y 30 de diciembre de 1938. 
Es importante destacar que la primera iniciativa de la posguerra para moderar la competencia en precios correspondió a la propia banca privada. En diciembre de 1940 se reunieron el Banco de Bilbao, el Banco de Castellón, el Banco Central, el Banco Español de Crédito, el Banco Guipuzcoano, el Banco Hispano Americano, el Banco Internacional de Industria y Comercio, el Banco de Valencia y el Banco de Vizcaya «con el fin de llegar a un acuerdo sobre diversos puntos de la actividad bancaria y evitar competencias entre los distintos establecimientos», según se dice en las instrucciones que se cursaron a continuación. Estas instrucciones eran un documento de 19 puntos que establecía la entrada en vigor del acuerdo el 1 de enero de 1941 para los tipos pasivos y el 15 del mismo mes para los activos 5 .

Como era de esperar, los tipos de interés indicados se consideraban máximos en el caso de los pasivos y mínimos en el de los activos, excepción hecha de los «créditos forzosos» o morosos que quedaban al margen del acuerdo. Los tipos pasivos suponían una reducción de medio punto porcentual con relación a los vigentes desde 1938 , mientras que los activos venían a coincidir con los aplicados por el Banco de España. La instrucción séptima extendía el acuerdo a las comisiones, de modo que en los descuentos se aplicarían las tarifas mínimas vigentes y en el resto de créditos el 1 por 1.000 trimestral. La instrucción novena llegaba a establecer mínimos en los tipos y las comisiones de los créditos a corporaciones, agrupaciones y organismos de carácter oficial, y la décima lo hacía en los concedidos a otros bancos y banqueros.

Los jefes de las sucursales eran invitados a establecer contacto con sus equivalentes en las otras entidades firmantes, advirtiendo que «si existen en esa plaza o próximas Bancos ajenos a este arreglo, procede que los incluidos en él analicen juntos la situación que pueda crearse y, si el resultado del análisis aconseja tomar alguna providencia especial, lo digan a sus respectivos organismos rectores (Dirección de Sucursales, etc.) »r. Toda la responsabilidad en el cumplimiento del acuerdo recaía sobre estos jefes de sucursales, quienes deberían enviar una declaración «destinada al alto Tribunal de sanciones Bancarias» (sic), jurando por su honor respetar el acuerdo y «denunciar a su Dirección Central las infracciones que pudiera presenciar o conocer». Precisamente este último punto fue el único del acuerdo que entendió conflictivo la Dirección General de Banca y Bolsa:

${ }^{5}$ «Nueva regulación de tipos de interés de la banca privada. Instrucciones», s. f., en $\mathrm{ACH}$, índice 1.043, legajo 29.579. 
el citado tribunal resultaba «inadmisible, pues no debe la Administración consentir se cree un Organismo que al margen de ella adopte decisiones de su exclusiva competencia» ${ }^{6}$.

En los primeros meses de 1941 se hicieron gestiones para extender el ámbito de aplicación del acuerdo y, el 19 de febrero, Pablo Garnica, como presidente del Comité Central de la Banca Española, escribió al director general de Banca y Bolsa indicándole que el día anterior se había alcanzado un «arreglo moderador de la competencia» entre numerosos bancos ${ }^{7}$. Garnica decía que deliberadamente se había prescindido de consignar en el acuerdo norma alguna sobre las comisiones «por entender las empresas firmantes que era deber suyo notificar tal extremo a esa Dirección General». En ese momento se comunicaba el deseo de unificar en el 1 por 1.000 trimestral las comisiones de los préstamos.

El acuerdo de febrero se había firmado en las mismas oficinas del Comité Central de la Banca (Marqués de Cubas, 4), por lo que resultaba claro el respaldo de la patronal ${ }^{8}$. Sin embargo, no se consiguió involucrar al conjunto del sistema bancario. Sólo 32 entidades, incluyendo tres banqueros, sobre un total de 114 bancos inscritos, se unieron al acuerdo en ese momento. Las entidades adheridas fueron: Banco de Aragón, Banco Asturiano de Industria y Comercio, Banco de Ávila, Banco de Bilbao, Matías Blanco Cobaleda, Banco Castellano, Banco de Castellón, Banco Central, Banco de Crédito de Zaragoza, Banco Español de Crédito, García Calamarte y Cía., Banco de Gijón, Banco Guipuzcoano, Banco Herrero, Banco Hispano Americano, Banco Internacional de Industria y Comercio, Banca López Quesada, Banco Mercantil, Banco Minero Industrial de Asturias, Banco del Oeste de España, Hijos de Manuel Rodríguez Acosta, Banco Sainz, Banco de San Sebastián, Banco de Santander, Banco de Tolosa, Banco de Torrelavega, Banco Urquijo de Guipúzcoa, Banco Urquijo Vascongado, Banco de Valencia, Banco de Vizcaya, Banco Zaragozano y Anacleto Carbajosa. Se contaba, por tanto, con el apoyo de la práctica totalidad de la banca nacional y una nutrida representación de la banca regional y local; también estaban una veterana casa de banca, la de Matías Blanco Cobaleda, que en 1957 se transformaría en el Banco de Salamanca, y

${ }^{6}$ «Cuadro comparativo de los tipos fijados en el convenio y los vigentes», s. f, en $\mathrm{ACH}$, indice 1.043, legajo 59.579.

7 Carta del presidente del Comité Central de la Banca Española al director general de Banca y Bolsa, de fecha 19 de febrero de 1941, en ACH, índice 1.043, legajo 29.579.

${ }^{8} \mathrm{El}$ texto completo de este «arreglo moderador de la competencia» puede verse en índice 1.043 , legajo 29.579 . 
otras dos que al poco tiempo perderían su independencia: la granadina Hijos de Manuel Rodríguez Acosta, absorbida en 1946 por el Banco Central, y la del banquero de Toro (Zamora) Anacleto Carbajosa, que pasó a formar parte del Banco de Bilbao en 1947.

La principal novedad del acuerdo de febrero de 1941 sobre el alcanzado en diciembre de 1940 estribaba en la creación de unos organismos de vigilancia mucho más sofisticados: 1) el Pleno de las entidades agrupadas; 2) el Comité Delegado; 3) la Junta de Vigilancia. El Pleno estaría integrado por una representación de todas y cada una de las entidades firmantes, y sería quien designase un Comité Delegado formado por seis representantes de las entidades que tuviesen, «necesariamente, su residencia en Madrid». Según el punto 14 del acuerdo, este Comité Delegado se atribuía amplios poderes para rectificar, modificar, ampliar o suspender el acuerdo y, además, convocaría al Pleno sólo «cuando a juicio de los señores que integran el Comité Delegado la importancia de la cuestión debatida así lo exija» (aunque el punto 17 exigía, al menos, una reunión al año). Finalmente, la Junta de Vigilancia quedaría constituida por tres profesionales independientes, un letrado, un profesor mercantil y un experto en el negocio bancario, cuyo nombramiento sería consensuado y cuya función consistiria principalmente en «resolver y fallar las denuncias que sobre posibles infracciones se produzcan». La sanción a aplicar consistiria en una advertencia privada o pública, dándola a conocer en este caso al resto de los bancos adheridos al acuerdo.

El mismo día que se informaba a la Dirección General de Banca y Bolsa, el Comité Central de la Banca Española envió una copia del acuerdo al resto de las instituciones bancarias, invitándolas a adherirse hasta el día 25 de febrero y así poder participar en la elección de vocales de la Junta de Vigilancia, que se celebraría el 1 de marzo. Se advertía que, pasado el plazo, la adhesión sería mucho más costosa, pues debería contar con el respaldo de la mayoría de los votos del Comité Delegado?.

El informe correspondiente del director general de Banca y Bolsa fue negativo. Empezaba diciendo que

en principio y en términos generales es de estimar que aunque el Convenio no puede considerase lesivo para el interés público bajo ninguno de sus aspectos, sino que más bien sigue en su desarrollo las normas generales que el Gobierno imprime a su política financiera, no parece apropiado que

${ }^{9}$ Carta-circular del Comité Central de la Banca Española, de fecha 19 de febrero de 1941, en ACH, índice 1.043, legajo 29.579. 
se deje a la iniciativa privada, aunque ésta venga respaldada por el Comité Central de la Banca Española, la consecución de estos objetivos.

El director general también se opuso al «mal efecto que produce» admitir la posibilidad de descubiertos en cuenta corriente y, sobre todo, a que se creasen órganos de vigilancia privados que invadiesen «la esfera del derecho público a quien está reservada por disposiciones legales la competencia en esta materia» ${ }^{10}$.

El 22 de marzo de 1941 se firmó un nuevo Arreglo moderador de la competencia bancaria, que incorporaba al texto las comisiones y las últimas adhesiones, pero que, ante la falta de apoyo oficial, tuvo un carácter estrictamente privado, por lo que sólo alcanzaba a las entidades firmantes. Este acuerdo fue actualizado en los años siguientes por el Comité Delegado, quien promovió en 1944 la publicación de una Nueva edición de las condiciones del arreglo moderador de la competencia bancaria ${ }^{11}$.

Finalmente, cabe añadir que lo que sí consiguió con este proceso el Comité Central de la Banca Española fue revisar al alza las tarifas de comisiones vigentes que, como hemos dicho, eran de 1936. Hasta el Consejo Superior de las Cámaras de Comercio, Industria y Navegación estuvo de acuerdo en que la elevación de los gastos generales hacía inevitable esta subida, por lo que no hubo problema en aprobar al efecto la Orden Ministerial de 24 de julio de $1941^{12}$. Esta Orden confirmó el poder de las Juntas Locales de Banca, que habían sido creadas en 1930 para vigilar la aplicación de las tarifas y estaban formadas por los propios banqueros, que ejercían así una suerte de autocontrol.

\section{EL ACUERDO INTERBANCARIO SOBRE INTERESES DE CUENTAS PASIVAS DE 1949}

En 1946, con motivo de la discusión del proyecto de la nueva Ley de Ordenación Bancaria, quedó claro que la patronal bancaria era partidaria de acordar en su seno máximos y mínimos para los tipos de interés y las comisiones y, luego, obtener su refrendo oficial. Lo que en ningún caso se admitiría era lo que amenazaba el apartado $c$ ) del artículo $41 \mathrm{del}$

${ }^{10}$ Notas mecanografiadas sobre «Convenio entre la banca privada, moderador de la competencia», s. f., en ACH, indice 1.043, legajo 29.579.

${ }^{11}$ Véase este texto, que apenas difiere del de 1941, en ACH. índice 1.043, legajo 29.579.

12 Véase el informe del Consejo Superior de las Cámaras de Comercio, Industria y Navegación, ẹ $\mathrm{ACH}$, índice 1.043, legajo 29.579. 
proyecto: que el Ministerio de Hacienda, previo informe del restablecido Consejo Superior Bancario, pudiese fijar exactamente todas las condiciones de las operaciones bancarias. A la banca le pareció tan irracional la redacción de este artículo que hasta pensó que se trataba de una «errata material», pues era «un exceso manifiesto que no ha podido estar en la mente de los autores del proyecto» ${ }^{13}$. Este y otros aspectos molestos para la banca fueron finalmente retirados, y la Ley de Ordenación Bancaria aprobada el último día de 1946 fue fiel a su preámbulo, donde se decía no pretender «ninguna innovación fundamental» ${ }^{14}$.

En el transcurso del debate anterior, el Comité Central de la Banca Española se dirigió al director general de Banca y Bolsa para rogar que

se proceda a declarar obligatorias para todos los Bancos operantes en España las condiciones que, privadamente y dentro de las limitaciones legales, tiene establecidas un importante grupo de Bancos en su organización denominada «Arreglo Moderador de la Competencia Bancaria». Estas condiciones se hallan ya desde hace largo tiempo rigiendo en la mayoría de los Bancos y sobre la base de las mismas vienen desempeñándose sus principales servicios, pero existe un grupo de Establecimientos bancarios que no las aceptan voluntariamente y con ello introducen un elemento de competencia que contribuye extraordinariamente a disminuir la eficacia de dichos acuerdos privados ${ }^{15}$.

Además, la patronal bancaria solicitaba «la unificación de todas las normas disciplinarias del crédito», de modo que también alcanzasen a las cajas de ahorros, y una nueva elevación de las comisiones ${ }^{16}$.

${ }^{13}$ Véase Pons (2001), p. 684, quien cita los informes que el Consejo Superior Bancario y el Banco de Vizcaya hicieron sobre este punto, y que se encuentran depositados en el Archivo Histórico del Banco de España.

${ }^{14}$ La mayor preocupación de la banca fueron los arts. 44 y 45 del proyecto, que facultaban al ministro de Hacienda para establecer depósitos obligatorios en el Banco de España por el 20 por 100 de los recursos ajenos. En el debate en las Cortes, la banca tuvo el apoyo de tres de las seis enmiendas que se presentaron, argumentando los procuradores que la medida podría traer depresión económica e inflación simultáneamente, pues cabría la posibilidad de que los bancos constituyesen los depósitos pignorando fondos públicos en el Banco de España. También se decía que los depósitos obligatorios provocarian «alarmas graves» entre la clientela. Véase ACH, índice 1.916, legajo 59.252.

15 Carta del presidente y del secretario accidental del Comité Central de la Banca Espanola al director general de Banca y Bolsa, de fecha 18 de octubre de 1946, en ACH, índice 807 , legajo 19.928 .

${ }^{16}$ En los primeros meses de 1945 se había intentado sin éxito que se actualizase la Orden de 1938 imponiendo a bancos y cajas de ahorros una rebaja en los tipos pasivos máximos. La contestación de la Dirección General de Banca y Bolsa fue favorable a que 
Hubo que esperar a 1949 para que, con la intervención del subgobernador del Banco de España y vicepresidente del Consejo Superior Bancario, Luis Sáez de Ibarra, se consiguiese, tras varios meses de gestiones, la firma de un nuevo acuerdo interbancario con base muy amplia. El acto tuvo lugar el 19 de noviembre y dio como resultado un texto denominado Acuerdo Interbancario sobre Intereses de Cuentas Pasivas. Lamentablemente, el texto no se halla en los archivos consultados y sólo lo que se recoge en España Económica y Financiera, de esa fecha, constituye la fuente disponible ${ }^{17}$. La publicación saludaba el acuerdo con todo tipo de parabienes, hablando de la necesidad de «poner el natural obstáculo a las extralimitaciones de la competencia». Destacaba en particular el hecho de que lo habían firmado «los representantes de más del 90 por 100 de la Banca española, calculándose que no ha de pasar mucho tiempo sin que se registre la adhesión de los Bancos que hoy todavía no figuran como firmantes», añadiéndose como significativo que los cinco grandes figuraban en primer lugar en el acuerdo ${ }^{18}$. Sin duda esto era lo más notorio, pues los tipos pasivos aprobados (entre el 0,5 por 100 de las cuentas corrientes a la vista y el 2,50 por 100 para las imposiciones a un año) no diferían de los del acuerdo de $1941^{19}$. La duración inicial del convenio se estableció en seis meses y la garantía de su cumplimiento estribaba en que el sub-

esa reducción se consiguiese simplemente a través de pactos como el vigente para algunos bancos privados desde 1941. Véase $\mathrm{ACH}$, índice 807, legajo 19.928 .

17 Sin embargo, el autor de este trabajo ha tenido acceso a la copia que se guarda en el Banco de Vitoria, que contiene las rúbricas del subgobernador del Banco de España y vicepresidente del Consejo Superior Bancario, Luis Sáez de Ibarra, junto a las del marqués de Aledo (Banco Hispano Americano y Banco de San Sebastián), Pablo de Garnica Echevarría (Banesto), Julio de Arteche Villabaso (Banco de Bilbao y Banco del Comercio), conde de Cadagua (Banco de Vizcaya), Ignacio Villalonga Villalba (Banco Central), Emilio Botín Sáenz de Sautuola (Banco de Santander), Eduardo Comas Pérez-Caballero (Banco Zaragozano), José Luis Bas Rivas (Banco de Aragón), Félix Millet Maristany (Banco Popular Español), barón de Viver (Banco Hispano Colonial), barón de Satrústegui (Banco Guipuzcoano), Ignacio Herrero Garralda (Banco Herrero), Antonio Noguera Bonora (Banco de Valencia), José María Rodríguez González (Banco de Gijón), José María Irurita Villanueva (Banco Asturiano de Industria y Comercio), Félix Alfaro Fournier (Banco de Vitoria) y Carlos López Quesada-Bourbón (Banca López Quesada). Los firmantes representaban a muchos otros bancos, aunque no se explicitan en el texto del acuerdo.

${ }^{18} \mathrm{El}$ art. $6^{\circ}$ del acuerdo decía que sólo no entraría en vigor en Galicia, Navarra, Logroño y Salamanca, mientras que algunas entidades locales no se adhirieran: el Banco Pastor y el de La Coruña, en el primer caso; el Crédito Navarro y La Vasconia, en el segundo, y el Banco Coca y la Banca Matías Blanco Cobaleda, en el tercero.

${ }_{19}$ Se consideraban al margen de estos tipos los saldos interbancarios y, curiosamente, las cuentas a plazo de un año a favor de clientes de las oficinas bancarias establecidas en Vitoria y en las demás plazas de la provincia de Álava, que podrian continuar devengando hasta el 3 por, 100 anual (art. $2 .^{\circ}$ ). 
gobernador Sáez de Ibarra debería ser informado de los incumplimientos, circunstancias particulares, nuevas adhesiones y posibles renuncias.

\section{LA ORDENACIÓN REGULADORA DE CONDICIONES BANCARIAS DE 1952}

El pacto de 1949 se limitó, por tanto, a los tipos pasivos, por lo que no pudo ser satisfactorio para el sector de la banca, encabezado por los más grandes, que buscaba un mayor grado de colusión. El Consejo Superior Bancario trabajó en los años siguientes para ampliar el pacto y, finalmente, el 1 de mayo de 1952, difundió a través de la Circular 397 un proyecto de Ordenación Reguladora de Condiciones Bancarias entre las entidades bancarias. En la reunión del 30 de junio de 1952, el Consejo hizo firme su apoyo a la ordenación, que contaba ya con la adhesión de la mayor parte de las instituciones implicadas. La fecha de entrada en vigor del acuerdo se fijó en el 10 de noviembre.

Si el acuerdo de 1949 había conseguido la mediación del Banco de España, el de 1952 contó con el apoyo del Ministerio de Hacienda, siendo el primero que obtenía el refrendo del poder político buscado por los banqueros desde el origen. Primero fue la Dirección General de Banca y Bolsa, que en circular de fecha 29 de noviembre mostró su apoyo público al pacto y solicitó que las Juntas Provinciales de Banca, creadas en mayo de ese año como sucesoras de las Juntas Locales, «puedan colaborar eficazmente a la más estricta observancia de la citada Ordenación» ${ }^{20}$. Más decisiva fue la Orden Ministerial de 9 de diciembre de 1952 por la que, dado que los tipos y comisiones pactados «resultan perfectamente compatibles con ellos (los tipos y comisiones legales vigentes ${ }^{2 \mathbf{i}}$ ), ya que son superiores a los legales en las operaciones activas e inferiores en las operaciones pasivas», se disponía autorizar la aplicación del convenio y que la Dirección General de Banca y Bolsa tuviese conocimiento de las infracciones cometidas $^{22}$. La Circular 446 del Consejo Superior Bancario, de

${ }^{20}$ «Circular comunicando instrucciones a los presidentes de las Juntas Provinciales de Banca sobre funcionamiento y actividades de las mismas», de fecha 29 de noviembre de 1952, en $\mathrm{ACH}$, índice 807, legajo 19.962.

${ }^{21}$ Los vigentes en ese momento eran: la Orden de 25 de noviembre de 1938, que fijó los tipos de interés máximos de las operaciones pasivas; la Orden de 24 de julio de 1941, que había establecido comisiones mínimas y que había sido parcialmente modificada el 10 de marzo de 1948 y el 11 de enero de 1952, y la Orden de 28 de enero de 1952, que había aprobado las comisiones para las operaciones con comitentes del extranjero.

22 «Circular trasladando una Orden comunicada de 9 de diciembre de 1952», de fecha de 11 de diciembre de 1952, en $\mathrm{ACH}$, índice 807, legajo 19.954. 
enero de 1953, dio publicidad de lo acordado, advirtiendo de la discriminación a que se vería sometida toda aquella entidad que manifestase una actitud de rebeldía.

El acuerdo de 1952 decía en su artículo primero obligar a «la totalidad de los Bancos y Banqueros operantes en España y plazas de su soberanía, inscritos en el Registro de la Banca privada de la Dirección General de Banca y Bolsa, más el Banco Exterior de España en sus operaciones con su clientela particular privada». La ordenación establecía intereses máximos pasivos (entre 0,50 y 3 por 100 , según modalidades), mientras que los préstamos y descuentos tenían tipos en torno al 5 por 100, al que había que sumar una comisión fija del 1 por 1.000 trimestral, en línea con las subidas experimentadas por los tipos del Banco de España en 1947. Las únicas excepciones eran los «créditos forzosos» (con dificultades del deudor), los anticipos al personal y los saldos entre instituciones financieras. Una Junta de Vigilancia (formada por banqueros) y otra Junta Superior (integrada por el director general de Banca y Bolsa, el subgobernador del banco de España y un miembro de la otra Junta) se encargarían del control del pacto. Las sanciones podrían llegar a obligar al banco a pagar diez veces su cuota de sostenimiento del Consejo Superior Bancario ${ }^{23}$.

\section{EL CONVENIO DE CONDICIONES BANCARIAS DE 1960}

El acuerdo interbancario de 1952 tan trabajosamente logrado fue un fracaso en la práctica, principalmente en lo que se refiere a intereses pasivos. Lo reconoce el Anteproyecto de Reglamentación Bancaria que realizaron en enero de 1960 , a requirimiento del director general de Banca, Bolsa e Inversiones, los banqueros Ignacio Villalonga y Luis de Usera, presidente del Banco Central y consejero delegado del Banco Hispano Americano, respectivamente:

La intensa competencia que en la actividad bancaria viene existiendo ha ido derivando, en parte, hacia el otorgamiento de intereses especiales a cuentas corrientes, cuentas de ahorro y cuentas a plazo [... hay] cuentas acreedoras que de modo más o menos encubierto vienen siendo liquidadas a tipos que triplican, cuadruplican y hasta, en ocasiones, decuplican aquéllos [...]. Todos

${ }^{23}$ El texto completo del convenio puede verse en $\mathrm{ACH}$, indice 807, legajo 19.954. 
aquellos pactos representaron nobles deseos de una mayoría, que no llegaron a tener completa realización ${ }^{24}$.

Para Villalonga y Usera, la solución pasaba por intensificar las sanciones, tratando de evitar así una intervención directa del poder político.

Del citado anteproyecto salió un Convenio de Condiciones Bancarias, aprobado en febrero de 1960, ante los «requerimientos apremiantes del Ilmo. Sr. Director General de Banca, Bolsa e Inversiones» para corregir «prácticas viciosas de otorgar intereses especiales a cuentas corrientes, cuentas de ahorro y cuentas a plazo y de aplicar en operaciones activas condiciones más favorables que las señaladas como mínimas obligatorias». El texto tenía 27 reglas, destacando: la primera, que establece que el documento tendrá «carácter de estrictamente reservado y se entregará para su custodia al Secretario del Consejo Superior Bancario»; la tercera, que establecía intereses máximos para los depósitos de todo tipo y vencimientos fijos para las imposiciones; la cuarta, que establecía intereses y comisiones mínimos para los descuentos y préstamos ${ }^{25}$; la quinta, que creaba Jurados de Primera Instancia de la Reglamentación Bancaria y Jurados de Apelación de la Reglamentación Bancaria; la diecinueve, que establecía «votación secreta por medio de bolas blancas y negras» para determinar la culpabilidad del denunciado; la veintiuna, que exigía la comunicación del veredicto al director general de Banca, Bolsa e Inversiones; la veintidós, que establecía como sanciones: multas, inversiones en fondos públicos, reducción del límite de redescuento, rebajas del dividendo a distribuir y la aplicación al banco sancionado en las transacciones interbancarias de las tarifas para la clientela de 1941; la veintisiete y última, que establecía la desaparición de la Junta de Vigilancia y la Junta Superior creadas por la ordenación reguladora de las condiciones bancarias de 30 de junio de $1952^{26}$. Este acuerdo de 1960 no llegó a plasmarse en una disposición legal.

${ }^{24}$ «Anteproyecto de reglamentación bancaria que presenta la ponencia designada al efecto, integrada por los señores D. Ignacio Villalonga Villalba y D. Luis de Usera López-González», enero 1960 , en $\mathrm{ACH}$, índice 807, legajo 19.954.

${ }^{25}$ En este caso, como en los demás, la banca siempre procuraba ampliar el margen financiero, de modo que, aunque era inevitable cierto ajuste a la situación del mercado monetario, se trataba claramente de mantener bajos los tipos pasivos y altos los activos.

${ }^{26}$ «Convenio de Condiciones Bancarias», febrero de 1960, en $\mathrm{ACH}$, indice 807 , legajo 19.928 . 


\section{EL CLUB DE LOS SIETE GRANDES}

En el otoño de 1964 el incumplimento del pacto de 1960 tuvo que ser notorio para que el Ministerio de Hacienda se decidiese a publicar la Orden de 10 de octubre que exigía en términos muy duros «el más exacto cumplimiento por la Banca privada, Cajas de Ahorro y Cajas Rurales de las normas vigentes sobre intereses y comisiones que dichas instituciones apliquen en sus operaciones activas y pasivas». La Inspección del Banco de España y del Instituto de Crédito de las Cajas de Ahorros se encargarían a partir de ahora de vigilar ese cumplimiento. Para aclarar qué tipos y comisiones eran los aplicables se dictaron el día 30 órdenes específicas para cada tipo de institución, que resultaron ser los de 1960 pero corregidos a la baja, en la misma dirección en que se había movido la política monetaria (cuadro 1). Fue la primera vez en que los tipos activos de la banca privada quedaban recogidos en el Boletín Oficial del Estado. La inflación obligó a que la tabla de tipos fuese actualizada en 1966 y 1967, como también lo fueron las comisiones hasta su completa liberalización en 1972.

\section{CUADRO 1}

Evolución de los tipos de interés de referencia para el sistema

bancario entre 1938 y 1964

(porcentajes)

\begin{tabular}{|c|c|c|c|c|c|c|}
\hline & 1938 & 1941 & 1949 & 1952 & 1960 & 1964 \\
\hline Cuentas corrientes .... & 1 & 0,5 & 0,5 & 0,5 & 0,5 & 0,5 \\
\hline Cuentas de ahorro .... & 2 & 2 & 2 & 2 & 2 & 2 \\
\hline $\begin{array}{l}\text { Imposiciones a plazo } \\
\text { fijo }\end{array}$ & De 2 a 3 & De 1,5 a 2,5 & De 1,5 a 2,5 & De 2,5 a 3 & De 2 a 3 & De 2 a 4 \\
\hline $\begin{array}{l}\text { Descuentos comer- } \\
\text { ciales......................... }\end{array}$ & & 4 & & $\mathrm{El}$ del BE & 6 & 5 \\
\hline $\begin{array}{l}\text { Descubiertos .............. } \\
\text { Créditos con garantía }\end{array}$ & & De 4,75 a 5 & & 6 & 7,25 & 7 \\
\hline 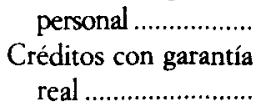 & & $\operatorname{De} 4$ a 4,5 & & De 4,5 a 5,25 & $\begin{array}{l}\text { De } 6 \text { a } 6,5 \\
\text { De } 6 \text { a } 6,25\end{array}$ & De 5 a 6 \\
\hline
\end{tabular}

Nota: $\mathrm{BE}=$ Banco de España. Se trata de tipos máximos para las operaciones pasivas (cuentas corrientes, cuentas de ahorro e imposiciones a plazo fijo) y de mínimos para las activas (descuentos comerciales, descubiertos, créditos con garantía personal y créditos con garantía real).

Fuente: Para 1938, Orden Ministerial de 25 de noviembre de 1938. Para 1941, 1949, 1952 y 1960, acuerdos interbancarios citados en el texto. Para 1964, Orden Ministerial de 30 de octubre de 1964 . 
Una Ley de 18 de marzo de 1966 alteró la redacción del art. 43 de la Ley de Ordenación Bancaria de 1946 (vigente en este punto) y consolidó la potestad del Ministerio de Hacienda para que, previo informe del Banco de España, el Consejo Superior Bancario, la Organización Sindical y el Consejo de Economía Nacional, pudiese estabablecer toda la gama de tipos activos y pasivos de las instituciones financieras. Buena prueba de que la liberalización financiera estaba siendo postergada con respecto a la liberalización del sector real ${ }^{27}$. Haciendo uso de este poder omnímodo sobre los precios, una Orden de 21 de julio de 1969 modificó el conjunto de los tipos de interés de la banca privada y del Banco Exterior de España, ligándolos mediante un diferencial al tipo de interés básico del Banco de España. Una novedad importante es que los tipos activos serían ahora máximos y no mínimos, por lo que había cambiado su función: ya no se trataba de eliminar la competencia, sino de establecer techos que abarataran el crédito y ayudasen, a través de la reducción de los costes financieros, al control de la inflación. También debe señalarse que los créditos a dos o más años quedaron libres. Este régimen de tipos de interés no fue alterado por la reforma liberalizadora de agosto de 1974, que simplemente los actualizó, como antes se había hecho en 1970,1971 y 1973 .

No existe constancia de nuevos «arreglos» interbancarios al estilo de los de 1941, 1949, 1952 y 1960. La estrategia seguida por la gran banca, que fue la que siempre propició este tipo de acuerdos pretendiendo disciplinar el sector, varió desde 1962. En abril de ese año, el presidente del Banco Español de Crédito, marqués de Deleitosa, pidió que los banqueros empeñasen su palabra de honor en contra de los «extratipos», definidos como «práctica viciosa» ${ }^{28}$. Por su parte, Ignacio Villalonga convenció a los presidentes de los mayores bancos para que se reuniesen mensualmente con el fin de coordinar sus actuaciones al margen del resto del sector ${ }^{29}$. Fruto de estas reuniones fue la propuesta

${ }^{27}$ Ésta es la tesis que se defiende en Lukauskas (1997). En García Ruiz (1992) se demuestra que esta graduación de la apertura, que sigue las tesis de R. MacKinnon, es clave para entender el éxito del caso español frente al fracaso de la Argentina de la dictadura que optó por una apertura total y acelerada.

${ }^{28}$ Actas del Consejo de Administración del Banco Español de Crédito de 29 de abril de 1962 y 29 de septiembre y 28 de octubre de 1964 .

29 Tortella (2000), p. 360 . De forma contradictoria, Villalonga fue un gran defensor del liberalismo radical de la Escuela Austríaca pero, en asuntos bancarios, siempre defendió la concertación, quizás porque el banco que presidía tenía fuertes intereses en el sector 
de una «fórmula normativa» (en expresión de Deleitosa) para acabar con los «extratipos», que el Ministerio de Hacienda terminó por plasmar en la citada Orden de octubre de 1964. Pero esta exigente regulación pronto fue sorteada por una incipiente «ingeniería financiera» que sacaba partido de las privilegiadas condiciones en que operaban los bancos industriales filiales o lanzaba productos imaginativos como las célebres «cuentas con seguro» del Banco de Vizcaya y del Banco de Santander de 1968.

En los años setenta, las reuniones se celebraron semanalmente a instancias de José María Aguirre Gonzalo, por ser el presidente de la entidad líder, el Banco Español de Crédito ${ }^{30}$. Fue el llamado «Club de los Siete Grandes» (pues pretendía englobar a quienes ocuparan los siete primeros puestos del ranking), donde, para evitar dar la sensación de actuar con secretismo, el gobernador del Banco de España era invitado con regularidad. En su seno se propiciaron los acuerdos de 1971, que ya sólo implicaron a la gran banca ${ }^{31}$. Dado que se trataba de un informal «pacto entre caballeros», no existe constancia documental de lo acordado en estas reuniones, pero con seguridad se hablaría de las reformas liberalizadoras de mediados de los años setenta que la banca recibió con inquietud. Enrique Fuentes Quintana, que era vicepresidente del Gobierno y máximo responsable económico, recuerda como «amarga» una reunión, a finales de 1977 , con representantes de los mayores bancos nacionales que consideraron -con pocas excepciones como Sánchez Asiaín, del Banco de Bilbao, o Termes, del Banco Popular - «peligrosa» e «inoportuna» la liberalización en curso ${ }^{32}$.

Alguna directriz emanada del Club llegó a la prensa, como la circular que aludía a la necesidad de cancelar todas las «condiciones especiales» a partir del 7 de abril de 1975, eliminar la disponibilidad anticipada de los saldos en imposiciones a plazo, prohibir la anticipación de los intereses y gravar con un 15 por 100 , en concepto de impuesto sobre las rentas de capital, todos los intereses devengados. También se decía que se debía advertir al cliente que estaba acordado por todos los bancos importantes

industrial y ello le llevaba a desear un marco institucional que asegurase mejor la estabilidad a largo plazo.

${ }^{30}$ Cuando el Banco Central encabezó el ranking, los almuerzos fueron organizados por su presidente, Alfonso Escámez.

31 Actas del Consejo de Administración del Banco Español de Crédito de 24 de febrero y 24 de noviembre de 1971 .

${ }^{32}$ Fuentes Quintana (1991), pp. LXII y LXIV. 
actuar de la misma manera, y se solicitaba de los gestores y directores que informaran a la superioridad de cualquier desviación sobre las normas observada en la competencia ${ }^{33}$. Poco después, las Órdenes Ministeriales de 23 de julio de 1977, que iniciaron la verdadera liberalización de los tipos de interés, empezando por las operaciones a plazo superior a un año, hicieron que muy pronto careciese de sentido hablar de «extratipos» ${ }^{34}$. Tras la crisis de los años ochenta y la entrada de España en la Unión Europea, que abría un horizonte de gran competitividad, la banca española dio por finalizada la fase oligopolística, que, como hemos visto, fue mucho más imperfecta de lo que muchas veces se supone.

\section{EL BANCO HISPANO AMERICANO FRENTE A LOS «ARREGLOS»INTERBANCARIOS}

Cuando en 1949 se alcanzó el primer gran acuerdo interbancario de la posguerra, el Banco Hispano Americano era todavía la entidad líder del sistema bancario español. El máximo directivo de este banco era su consejero delegado, Andrés Moreno, un hombre formado en la tradición liberal y conservadora de la banca inglesa, que no creía en la banca mixta (de ahí el famoso «Pacto de las Jarillas» con el Banco Urquijo que le permitió ceder a esta entidad las iniciativas industriales) ni en la formación de cárteles ${ }^{35}$. Por ello, no es extraño que hayamos encontrado pruebas de que el Banco Hispano Americano abandonó muy pronto la disciplina del acuerdo de 1949, sobre el que públicamente Andrés Moreno había mostrado sus recelos, ofreciendo «extratipos», es decir, tipos de remuneración del pasivo superiores a lo establecido legalmente ${ }^{36}$.

Los «extratipos» empezaron siendo cifras muy modestas, pero a partir de 1952 crecieron hasta llegar a representar en 1959 una cifra equivalente a una tercera parte del beneficio neto. Los datos se exponen en el cuadro 2, donde aparece un estudio paralelo de comisiones y «extratipos», pues una parte importante de éstos se financiaba con aquéllas.

33 Ver el texto completo de la circular en Doblón, junio de 1975, p. 7.

34 Todavía en junio de 1980 se quiso proponer un acuerdo para limitar los tipos pasivos, pero incluso Aguirre Gonzalo no podía sino reconocer que «todo lo que se acordase en este terreno estaba condenado al incumplimiento». Acta del Consejo de Administración del Banco Español de Crédito de 18 de junio de 1980.

${ }^{35}$ Véase García Ruiz (2000).

36 Véase Tortella y García Ruiz (1999), pp. 253-256 y 378-381. 


\section{CUADRO 2}

Evolución de los pagos por comisiones y «extratipos» del Banco Hispano Americano, 1949-1960

(millones $y$ porcentajes)

\begin{tabular}{|c|c|c|c|c|}
\hline & $\begin{array}{c}\text { Saldo neto } \\
\text { de comisiones }\end{array}$ & $\begin{array}{c}\text { Pagos } \\
\text { por «extratipos» }\end{array}$ & $\begin{array}{c}\text { Saldo neto } \\
\text { de comisiones/ } \\
\text { Beneficio neto } \\
\text { (\%) }\end{array}$ & $\begin{array}{c}\text { Pagos } \\
\text { por «extratipos»/ } \\
\text { Beneficio neto } \\
(\%)\end{array}$ \\
\hline 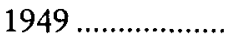 & 109 & 95 & 1 & 1 \\
\hline $1950 \ldots \ldots \ldots \ldots \ldots$ & 135 & 111 & 6 & 5 \\
\hline $1951 \ldots \ldots \ldots \ldots \ldots$ & 175 & 136 & 7 & 6 \\
\hline $1952 \ldots \ldots \ldots \ldots \ldots \ldots$ & 198 & 132 & 23 & 16 \\
\hline $1953 \ldots \ldots \ldots \ldots \ldots$ & 220 & 129 & 8 & 5 \\
\hline 1954 .................. & 129 & 77 & 29 & 17 \\
\hline $1955 \ldots \ldots \ldots \ldots \ldots$ & 177 & 74 & 50 & 21 \\
\hline $1956 \ldots \ldots \ldots \ldots \ldots$ & 248 & 96 & 61 & 24 \\
\hline $1957 \ldots \ldots \ldots \ldots \ldots$ & 297 & 110 & 79 & 29 \\
\hline $1958 \ldots \ldots \ldots \ldots \ldots$ & 392 & 85 & 91 & 20 \\
\hline 1959 .................... & 402 & 83 & 161 & 33 \\
\hline $1960 \ldots \ldots \ldots \ldots \ldots$ & 491 & 97 & 70 & 14 \\
\hline
\end{tabular}

FuENTE: Tortella y García Ruiz (1999), p. 254.

En 1953 empezó a inquietar el «ritmo acelerado con que se producen liquidaciones con intereses diferenciales ["extratipos"]», como se dice en los Informes de Contabilidad General, que ha sido la fuente del estudio. Fruto de esa preocupación fue el seguimiento que se hizo entre 1954 y 1959 del «rendimiento de los "extratipos"», es decir, de su influencia en el aumento efectivo de la captación de recursos ajenos (cuadro 3). El sobrecoste medio impuesto por los «extratipos» resultó especialmente alto en Cataluña $(0,54$ por 100$)$ y Galicia $(0,41)$, seguidas a distancia por Madrid $(0,27)$ y el resto. Las características socioeconómicas de Cataluña y Galicia eran - y sontan dispares que sólo cabe pensar en que el ofrecimiento de «extratipos» era establecido de forma absolutamente casuística y circunstancial.

¿Hubo resultados? Un sencillo análisis indica que el coeficiente de correlación entre sobrecoste por «extratipos» e incremento de saldos acreedores es positivo, pero bajo: 0,37 . En Cataluña y Galicia se acrecentaron los saldos medios por encima del 13 por 100 , pero porcentajes similares se alcanzaron en Extremadura o Castilla la Vieja con sobrecostes muy inferiores; en Madrid el crecimiento fue superior al 14,5 por 100 , y en Canarias al 15,50 por 
CUADRO 3

Incidencia de los «extratipos» en la atracción de recursos ajenos para el Banco Hispano Hispano Americano, 1954-1959

(porcentajes)

\begin{tabular}{|c|c|c|c|c|c|}
\hline & $\begin{array}{c}\text { Sobrecoste } \\
\text { medio }\end{array}$ & $\begin{array}{l}\text { Crecimiento } \\
\text { medio de los } \\
\text { recursos }\end{array}$ & & $\begin{array}{c}\text { Sobrecoste } \\
\text { medio }\end{array}$ & $\begin{array}{l}\text { Crecimiento } \\
\text { medio de los } \\
\text { recursos }\end{array}$ \\
\hline Badajoz ................. & 0,23 & 13,98 & Málaga................ & 0,28 & 9,72 \\
\hline Barcelona ............... & 0,54 & 13,82 & Murcia ............... & 0,20 & 8,86 \\
\hline Bilbao .................... & 0,10 & 13,50 & Salamanca .......... & 0,08 & 10,71 \\
\hline Ceuta/Tetuán....... & 0,07 & $-12,86$ & Sevilla ................ & 0,35 & 11,96 \\
\hline Granada............... & 0,19 & 11,96 & Valencia............. & 0,22 & 12,05 \\
\hline La Coruña ............ & 0,41 & 13,41 & Valladolid ........... & 0,10 & 13,32 \\
\hline Las Palmas............ & 0,00 & 15,67 & Zaragoza ............ & 0,17 & 15,26 \\
\hline Madrid ................... & 0,27 & 14,60 & Media ................. & 0,29 & 13,54 \\
\hline
\end{tabular}

- Nota: Se citan por orden alfabético las sedes de las Direcciones Regionales del banco. FueNTE: Tortella y García Ruiz (1999), p. 255.

100 con un sobrecoste nulo. En definitiva, el pago de mayores tipos de interés distaba de implicar un aumento proporcional de los depósitos.

El problema es que una vez iniciada la competencia por la vía de los «extratipos» resultaba cada vez más peligroso descolgarse. Con seguridad, habría un desplome de los depósitos si se actuaba de forma unilateral. El Banco Hispano Americano se encontraba ante un problema de elección similar al conocido como «dilema del prisionero» ${ }^{37}$. Pero los costes se disparaban y la preocupación aumentaba sin cesar: «crecido coste, en progresión alarmante» (1956), «crecimiento desaforado» (1957) y expresiones similares se leen en los Informes de Contabilidad General. Al fin, con el acuerdo de febrero de 1960, que entró en vigor el 10 de abril, llegó la solución, que no podía ser otra sino un nuevo acuerdo interbancario para limitar la competencia en tipos de interés ${ }^{38}$. El Informe de Contabilidad

${ }^{37} \mathrm{El}$ «dilema del prisionero» es un viejo y conocido juego que se estudia mucho en ciencias sociales, por el que que dos prisioneros descubren que es mejor apoyarse entre sí que denunciar al otro. El juego admite numerosas formulaciones, pero siempre llega a la conclusión de que la cooperación es mejor que la actuación unilateral.

${ }^{38}$ Que el oligopolio bancario es rentable parece probado. Jones (1993), p. 388, afirma que la banca inglesa obtuvo mayores beneficios a largo plazo en las actividades internas que en las externas, principalmente durante la fase oligopolística que tuvo lugar entre la Primera Guerra Mundial y los inicios de los años sesenta. 
General de ese año mostró su satisfacción porque «el crecimiento de los recursos ajenos, iniciado ya en los últimos meses de 1959, prosiguió durante todo el año 1960 de una manera extraordinaria, especialmente, en las posiciones más rentables como consecuencia de la desaparición en abril de los tipos de interés excepcionales ("extratipos")».

El sucesor de Andrés Moreno, fallecido en enero de 1960, fue Luis de Usera, un hombre que mantendría actitudes más cooperativas, como lo prueba el informe antes citado que redactó junto a Ignacio Villalonga, un firme partidario de la colusión. Sin embargo, en la práctica, los datos disponibles nos dicen con claridad que el Banco Hispano Americano siguió saliéndose de las normas establecidas, como posiblemente lo hacían el resto de las instituciones financieras. A la vista del cuadro 4, es comprensible el enfado que traslucen las Órdenes Ministeriales de 1964 o el intento de poner límites de la circular bancaria de 1975 (evidentemente no cumplida).

\section{CUADRO 4}

Evolución de los pagos por comisiones y «extratipos»

del Banco Hispano Americano, 1961-1980

(millones y porcentajes)

\begin{tabular}{|c|c|c|c|c|}
\hline & $\begin{array}{c}\text { Saldo neto } \\
\text { de comisiones }\end{array}$ & $\begin{array}{c}\text { Pagos } \\
\text { por wextratipos» }\end{array}$ & $\begin{array}{c}\text { Saldo neto } \\
\text { de comisiones } \\
\text { Beneficio neto } \\
\text { (\%) }\end{array}$ & $\begin{array}{c}\text { Pagos } \\
\text { por «extratiposw } \\
\text { Beneficio neto } \\
(\%)\end{array}$ \\
\hline $1961 \ldots \ldots \ldots \ldots \ldots$ & 568 & 0 & 106 & 0 \\
\hline $1962 \ldots \ldots \ldots \ldots \ldots \ldots$ & 590 & 8 & 102 & 1 \\
\hline $1963 \ldots \ldots \ldots \ldots \ldots$ & 803 & 50 & 130 & 8 \\
\hline $1964 \ldots \ldots \ldots \ldots \ldots$ & 941 & 157 & 145 & 24 \\
\hline $1965 \ldots \ldots \ldots \ldots \ldots$ & 1.161 & 0 & 147 & 0 \\
\hline $1966 \ldots \ldots \ldots \ldots \ldots$ & 1.490 & 0 & 165 & 0 \\
\hline $1967 \ldots \ldots \ldots \ldots \ldots$ & 1.477 & 0 & 138 & 0 \\
\hline $1975 \ldots \ldots \ldots \ldots \ldots$ & 6.326 & 1.425 & 130 & 29 \\
\hline $1976 \ldots \ldots \ldots \ldots \ldots$ & 8.382 & 1.780 & 144 & 31 \\
\hline $1977 \ldots \ldots \ldots \ldots \ldots$ & 14.376 & 2.236 & 236 & 37 \\
\hline $1978 \ldots \ldots \ldots \ldots \ldots$ & 19.734 & 82 & 284 & 1 \\
\hline 1979 ................... & 32.982 & 3 & 412 & 0 \\
\hline $1980 \ldots \ldots \ldots \ldots \ldots$ & 34.694 & 0 & 361 & 0 \\
\hline
\end{tabular}

Nota: No se dispone de datos entre 1968 y 1974.

FUENTE: Tortella y García Ruiz (1999), p. 381. 
En la fuente no se dispone de información para el período 1968-1974, pero podemos imaginar una evolución al alza de los «extratipos» desde el momento en que los datos sobre tipos de interés declarados contenidos en Cifras y relaciones significativas de los balances de la banca privada, publicación impulsada entre 1968 y 1985 por el Consejo Superior Bancario, muestran que, progresivamente, los bancos de ámbito nacional tendieron a practicar políticas propias y no coordinadas desde principios de la década de 1970. Con todo, la conducta oligopolística parecía ser menor en la banca regional, local o industrial, a juzgar por la mayor diversidad en los tipos declarados.

\section{CONCLUSIONES}

En este trabajo se ha demostrado que los bancos españoles - principalmente, sus grandes entidades, que acumulaban hasta dos terceras partes de los depósitos bancarios en los años cuarenta y cincuenta- siguieron durante el franquismo su tendencia a la colusión, que venía al menos de la época de la Primera Guerra Mundial, cuando se constituyeron las primeras patronales. Doce años tuvieron que pasar, desde diciembre de 1940 hasta diciembre de 1952, para que el Estado se animase a sancionar oficialmente los pactos buscados ansiosamente por la gran banca como forma de disciplinar al resto del sistema. El Gobierno parecía contentarse con la Orden de 1938 que, haciendo uso de las atribuciones que le confería la Ley de Ordenación Bancaria de 1921, estableció tipos pasivos máximos. Hasta 1964 no se publicó en el Boletín Oficial del Estado toda la gama de tipos, máximos para los pasivos y mínimos para los activos. Poco después, en 1969, el Gobierno cambió su política y decidió que los tipos activos oficiales no fueran mínimos sino máximos. Con ello, dejaba de respaldar el oligopolio y buscaba establecer una contención de los costes financieros para las empresas, objetivo más razonable desde el punto de vista de la política económica. Al mismo tiempo se iniciaba la liberalización por las operaciones a largo plazo. Por tanto, se puede concluir que hubo muchos roces y desencuentros entre el Estado y la gran banca en el proceso de formación de los precios del sistema financiero durante el franquismo.

Por otra parte, el incumplimiento de los acuerdos queda probado para el Banco Hispano Americano, tanto en la época de Andrés Moreno, que por su formación liberal siempre desconfió de estos «arreglos», como en la de Luis de Usera, más partidario de la colusión. El carácter de banco 
comercial que siempre tuvo el Hispano Americano ayuda a entender su posición, que contrasta con la de bancos más implicados con la industria, como el Español de Crédito o el Central, que precisaban operar en un marco de estabilidad a medio y largo plazo y se constituyeron en máximos defensores de la concertación. En cualquier caso, el comportamiento del Banco Hispano Americano no puede considerarse excepcional en absoluto y viene a confirmar lo que Pons (2001) ha encontrado en sus estimaciones para el conjunto del sistema bancario.

En resumen, los documentos aportados en esta investigación permiten conocer con mayor precisión lo que ocurrió durante el franquismo con la regulación de los tipos de interés y comisiones de las operaciones de depósito y crédito, y poner en duda que el oligopolio bancario funcionase de forma tan rigurosa y exacta como supusieron sus críticos de los años cincuenta y sesenta. Por debajo de las apariencias y de los intentos por controlar el mercado hubo más competencia de lo que se ha venido admitiendo.

\section{BIBLIOGRAFÍA}

Consejo Superior BanCario (1968-1985): Cifras y relaciones significativas de los balances de la banca privada, Madrid.

- (1982): Recopilación de circulares, 1947-1981, Madrid.

Fuentes Quintana, E. (1991): «Prólogo», en Termes, R.: Desde la banca, volumen 1, Madrid, Rialp, pp. LI a XCVII.

GARCIA RUIZ, J. L. (1992): «Sector financiero y apertura económica: una perspectiva comparada entre la Argentina y España», volumen 2, Ciclos, núm. 3, pp. 95-112.

- (2000): «Andrés Moreno García (1895-1960)», en TORREs, E. (dir.): Los 100 empresarios españoles del siglo xx, Madrid, LID, pp. 350-353.

JONES, G. (1993): British multinational banking, 1830-1990, Oxford, Clarendon Press.

LuKauskas, A. (1997): Regulating Finance: The Political Economy of Spanish Financial Policy from Franco to Democracy, Ann Arbor, The University of Michigan Press.

Martín Aceña, P., y Pons, M. A. (1994); «Spanish banking after the Civil War, 1940-1962», Financial History Review, volumen 1, núm. 2, pp. 121-138.

Muñoz, J. (1969): El poder de la banca en España, Algorta, Zero.

Pérez, S. (1997): Banking on Privilege. The Politics of Spanish Financial Reform, Ithaca, Cornell University Press.

Pons, M. A. (2001): «Oligopolio y tipos de interés en la banca española, 1942-1975», en Revista de Historia Económica, volumen 19, núm. 3, pp. 679-703.

- (2002): Regulating Spanish Banking, 1939-1975, Aldershot, Ashgate. 
TEDDE, P. (2001): «El sistema financiero en la España del siglo XX», en AA. VV.: Las claves de la España del siglo XX. Las transformaciones económicas, Madrid, Sociedad Estatal España Nuevo Milenio, pp. 141-184.

TERMES, R. (1991): Desde la banca, 2 vols., Madrid, Rialp.

TORTELlA, G. (2000): «Ignacio Villalonga Villalba (1895-1973)», en TORRES, E. (dir.): Los 100 empresarios españoles del siglo XX, Madrid, LID, pp. 354-360. Tortella, G., y Garcí Ruiz, J. L. (1999): Una bistoria de los Bancos Central e Hispano Americano (1900-2000). Un siglo de gran banca en España. Texto mecanografiado sin publicar.

- (2000): Banca y politica durante el primer franquismo. Ponencia presentada al Seminario «Política y Empresa en España, 1936-1957», Universidad de Alicante, 22-24 de noviembre.

- (2001): Spanish Banking after the Civil War: A Halting Reconstruction under Fascism. Ponencia presentada al Seminario «Crisis and Renewal in $20^{\text {th }}$ Century Banking», European Association for Banking History (EABH) Conference, Liubliana (Eslovenia), 18-19 de mayo.

\section{FUENTES}

No impresas

Archivo del Banco Español de Crédito.

Actas del Consejo de Administración.

Archivo Central de Hacienda (ACH).

Índice 807, legajos 19.928, 19.954 y 19.962.

Índice 1.043 , legajo 29.579.

Índice 1.916, legajo 59.252.

Impresas

Doblón.

España Económica y Financiera. 